\title{
基于根系发育分级的砂壤土下成熟林木根系构型 分析
}

\section{祝 维 ${ }^{1}$ 余立璇 ${ }^{1}$ 赵德海 ${ }^{2}$ 贾黎明 $1^{*}$}

${ }^{1}$ 北京林业大学省部共建森林培育与保护教育部重点实验室, 北京 $100083 ;{ }^{2}$ Warnell School of Forestry and Natural Resources, The University of Georgia, Athens, GA 30602, USA

摘 要 基于根系发育分级标准分析不同等级根系的形态特征和各级根系的生长发育策略, 可以为未来成熟林木根系预测 和模型构建提供参考。该研究以30多年生的两个毛白杨(Populus tomentosa)和一个刺槐(Robinia pseudoacacia)的根系系统为研 究对象, 通过全挖法获取研究材料, 以Rose (1983)提出的发育分级标准作为根系分级标准, 分析了不同等级根系的基径、根 长、连结长度、根系数量等形态特征以及不同等级根系间的拓扑关系。研究结果表明: 1) 3 个根系系统的修正拓扑指数 $q_{a} 、 q_{b}$ 均接近于 0 , 拓扑指数 $T I$ 均接近于 0.5 , 说明 3 个根系系统均呈现叉状分支; 3 个根系系统垂直分布最深达到 $5.7-6.4 \mathrm{~m}$, 水平分布 最长达到7.6-13.5 m; 同一树种根系存在连生关系。2) 3个根系系统能够分支到7-8级根; 一级根基径和长度显著高于后几级 根, 一级根基径是后几级根的5.79-36.92倍, 一级根长度是后几级根的1.45-9.11倍; 根系数量随着根系等级增加先增后减, 在 三级根上达到最大值。3)在前三级根中, 各级根上的连结长度从根基到根尖变化不大, 说明子级根在母级根上分布均匀, 能 够充分高效地吸收土壤资源。4)母级根对子级根的基径拟合线性方程斜率，一级根最小(平均斜率0.15), 二级根和三级根的斜 率相差不大(平均斜率 $0.34 、 0.35$ ), 说明一级根优先发育自身直径, 达到针固和支撑的作用, 二、三级根则会优先发育子级根, 通过不断增强子级根以达到高效占领土壤空间的目的。5)基径对长度的拟合线性方程斜率随着根系等级增加而增加(平均斜 率从10.46增长至90.43), 说明高级别根系会倾向于发展根系长度来达到探索资源、拓展空间的目的。

关键词 发育分级标准; 根系分级; 根系构型; 成熟林木; 砂壤土

祝维, 余立璇, 赵德海, 贾黎明 (2019). 基于根系发育分级的砂壤土下成熟林木根系构型分析. 植物生态学报, 43, 119-130. DOI: 10.17521/cjpe.2018.0269

\section{Architectural analysis of root systems of mature trees in sandy loam soils using the root development classification}

ZHU Wei ${ }^{1}$, YU Li-Xuan ${ }^{1}$, ZHAO De-Hai ${ }^{2}$, and JIA Li-Ming ${ }^{1 *}$

${ }^{1}$ Ministry of Education Key Laboratory of Silviculture and Conservation, Beijing Forestry University, Beijing 100083, China; ${ }^{2}$ Warnell School of Forestry and Natural Resources, The University of Georgia, Athens, GA 30602, USA

\begin{abstract}
Aims Understanding the differences in root architectural strategies among the species and the differences in morphological characteristics among different root orders will facilitate our understanding root growth and development strategies, and thus provide a basis for predicting and modeling the root systems for mature trees. In this study, we analyzed the morphological characteristics and topological relationships for the root systems of two Populus tomentosa trees and one Robinia pseudoacacia tree.

Methods A method combining both excavation and analysis was applied to extract and quantify root architectural characteristics of the three root systems. The morphological characteristics such as root basal diameter, root length, link length, and root number of different root orders were described using the developmental analysis method of Rose (1983), and their topological relationships were analyzed.

Important findings 1) The modified topological indices $q_{a}$ and $q_{b}$ were close to 0 , and the topological index TI was close to 0.5 for all three root systems, indicating their dichotomous structure. The depth and width of the systems ranged from 5.7 to $6.4 \mathrm{~m}$ and from 7.6 to $13.5 \mathrm{~m}$, respectively. Root grafts occurred in the same species. 2) The root systems could have the seventh or eighth order roots. The basal root diameter and root length significantly

收稿日期Received: 2018-10-30 接受日期Accepted: 2019-01-30

基金项目: 国家重点研发计划(2016YFD0600403)。Supported by the National Key R\&D Program of China (2016YFD0600403).

* 通信作者Corresponding author (jlm@bjfu.edu.cn)
\end{abstract}


decreased with increasing root order. The first order roots had 5.79-36.92 times the basal diameter and 1.45-9.11 times the length of higher order roots. With increasing root order, the root number increased, and reached a maximum value for the third order roots, and then decreased. 3) In roots of each of the first three orders, the link length varied little from the root base towards its tip, indicating that the child roots were distributed evenly on their parent roots and thus help trees absorb soil resources more efficiently. 4) The regression of basal diameters of child roots on basal diameters of their mother roots showed that the smallest slope for the first order roots (average slope 0.15$)$ and no big difference in the slope between the second and third order roots (0.34 versus 0.35$)$. This suggested that the first order roots developed their own diameter first for anchoring and supporting the tree, while the second and third order roots developed their child roots to facilitate nutrient uptake from the soil. 5) The regression of root length on root basal diameter suggested that the slope increased from 10.46 to 90.43 with increasing root order, which implies that the higher order roots tended to develop their length to explore resources and expand their space.

Key words developmental classification standard; root system classification; root architecture; mature tree; sandy loam soil

Zhu W, Yu LX, Zhao DH, Jia LM (2019). Architectural analysis of root systems of mature trees in sandy loam soils using the root development classification. Chinese Journal of Plant Ecology, 43, 119-130. DOI: 10.17521/cjpe.2018.0269

根系是树木的重要器官, 是树木生长发育的基 础。根系构型是指根系在生长介质中的空间排列和 分布(Lynch, 1995), 结构根的框架定义了吸收根的 位置, 从而定义了水和养分的吸收与传导(Lynch, 1995; Pagès et al., 2000)。具体来说，根系构型包括 立体几何构型和平面几何构型(严小龙等, 2000)。根 系构型对林木的稳定性、针定能力、水分养分运移 以及根系竞争等关键的生态过程起着重要作用 (Fitter, 1987; Lynch, 1995; Danjon et al., 2008)。

对根系构型的精确评估需要挖掘根部甚至整个 根系统, 这是昂贵且耗时的(Smit et al., 2000; Danjon \& Reubens, 2008)。这就是在根系构型的详细研 究中研究个体数量较少的原因(Vercambre et al., 2003; Danjon et al., 2005, 2013; Wagner et al., 2011; Valdes-Rodriguez et al., 2013), 而对成熟林木的根系 研究相对更少。有学者们使用三维仪器和软件来深 入探究成熟林木尤其是相当大的根系的结构与功能 (Danjon \& Reubens, 2008), 比如Gärtner等(2009)提 出用三维激光扫描(TLS)技术来为成熟云杉(Picea abies) 林木根系建立三维模型, 为根系针固研究和 根系年轮研究奠定基础; Danjon等(2005)使用3D数 字化设备对海岸松(Pinus pinaster)成熟林木的根系 结构和抗风力进行探究; 还有学者用吊车获取了 10 个不同树种的 106 个成熟林木根系系统, 揭示根系 构型中物种、环境、根大小和根类型之间的关系 (Vennetier et al., 2015); Osawa等(2010)在西伯利亚 多年冻土区通过挖掘法获取了不同林龄(幼龄林、成 熟林和过熟林)的落叶松(Larix gmelinii)树根, 研究
了完整根系的空间结构、根系分布与冠层分布的关 系以及根系结构与土壤因子的关系。成熟林木的完 整根系系统中各级根系间的几何形态和拓扑结构 对根系结构-功能的研究具有重要意义, 但是因为 工作复杂且繁琐, 至今没有研究深入和详细地探 索过。

根系分级一般有三种: 一种是依赖于发育模型 (Rose, 1983), 其中来自茎干基部的那些根被称为轴, 连续地承载更高阶的侧向根; 一种是从形态角度出 发, 基于Strahler (1957)的地貌系统, 颠倒了分类方 向, 因此所有终端分支都是一级, 2 个一级根的融合 产生二级根, 以此类推; 还有一种是基于连结的 (Fitter, 1986), 每个“连结” (两个节点之间的根长度) 被赋予其自己的身份, 随着根系系统的发展逐步变 化, 从而反映不断变化的生理功能。近 20 年来对于 细根不同根序根系的形态和功能研究甚多。自从 Pregitzer等(2002)关于北美9个树种根系系统尖端前 三级 (以第二种分级标准定义的)根序之间形态指 标、碳 $(\mathrm{C})$ 含量、氮 $(\mathrm{N})$ 含量的差异的研究报道后, 国 内外开始出现各种从根序角度探究细根形态和功能 的报道(常文静和郭大立，2008; Guo et al., 2008; Salahuddin et al., 2018)。显然不同根序尺度上根尖 前几级根系形态与功能的研究已经非常透彻, 但是 除根尖以外的整个根系系统的相关研究却非常少。

在采用全挖法研究林木的完整根系构型过程中, 由于成熟林木庞大的根系系统很难获取末端根系, 采用细根分级方法(即形态分级方法(Pregitzer et al., 2002))会导致分级混乱。因此, 我们以Rose (1983)

www.plant-ecology.com 
的发育分级标准作为本研究的根系分级标准。毛白 杨(Populus tomentosa)和刺槐(Robinia pseudoacacia) 是华北黄泛平原砂壤土立地上的主要造林树种, 以 它们成熟林木的根系系统为研究对象, 采用全挖法 获取根系材料, 通过寻找 3 个完整根系系统的构型 共同性, 来解答以下两个科学问题: (1)在发育分级 标准的前提下, 成熟林木完整根系系统各级根系形 态特征如何(即结构分析)? (2)在发育分级标准中, 各级根系的生长发育策略如何(即功能分析)?

\section{1 材料和方法}

\section{1 研究地概况}

研究地位于山东省高唐县国有旧城林场 $\left(36.81^{\circ}\right.$ $\mathrm{N}, 116.09^{\circ} \mathrm{E}$ ), 海拔 $30 \mathrm{~m}$, 属于黄泛冲积平原。该地 属暖温带半干旱季风区域大陆性气候, 具有显著的 季节变化, 光照充足, 热量丰富。年降水量 544.7 $\mathrm{mm}$, 降水主要集中在7-8月, 年蒸发量 $1880 \mathrm{~mm}$, 年平均气温 $12.0-14.1{ }^{\circ} \mathrm{C}$, 极端最高气温 $41.2{ }^{\circ} \mathrm{C}$, 极端最低气温 $-20.8{ }^{\circ} \mathrm{C}$ 。年日照时间达 $2651.9 \mathrm{~h}$, 无 霜期 204 天, 地下水位 $6 \mathrm{~m}$ 左右, 土壤质地为砂壤土 (砂粒含量65\%)。

在相邻的两片纯林和混交林内(表1)分别设定3 个 $30 \mathrm{~m} \times 30 \mathrm{~m}$ 样地，根据样地的平均树高及平均胸 径选取平均标准木。在纯林内选择1株毛白杨平均标 准木(基径 $42.9 \mathrm{~cm}$, 胸径 $35.0 \mathrm{~cm}$, 树高 $30.5 \mathrm{~m}$ ), 混 交林内选择相邻的 1 株毛白杨(基径 $37.2 \mathrm{~cm}$, 胸径 $35.9 \mathrm{~cm}$, 树高 $27.8 \mathrm{~m}$ )与 1 株刺槐(基径 $31.6 \mathrm{~cm}$, 胸径 $25.1 \mathrm{~cm}$, 树高 $20.8 \mathrm{~m}$ )。通过基径处的年轮数得知纯 林内毛白杨的树龄为 38 年, 混交林内毛白杨的树龄 为 35 年，混交林内刺槐的树龄为 34 年。

\section{2 根系挖掘方法}

伐去所选取的平均标准木的地上部分, 使用铁 锹、小铲子、螺丝刀等工具, 对平均标准木的根系 平均生长空间进行完全挖掘, 尽量保证整个根系系 统的完整性和空间性。纯林内毛白杨的主体挖掘范 围为直径 $6 \mathrm{~m}$ 的圆形大坑, 平均垂直深度 $1.5 \mathrm{~m}$; 混 交林内相邻的毛白杨和刺槐采取同时挖掘的方式, 挖掘范围为长 $7.5 \mathrm{~m}$ 、宽 $5.5 \mathrm{~m}$ 的方形大坑, 平均垂直 深度 $1.5 \mathrm{~m}$ 。3个根系系统完全挖掘出来后, 每个根 系系统在水平方向和垂直方向分别选择3-5个根系 进行追踪式挖掘, 直至挖掘到终点。3个根系系统分 别设定的挖掘空间范围能观测到研究目标树几乎所 有的根系系统。

\section{3 根系参数测定与数据分析}

本研究以Rose (1983)提出的发育分级标准为根 系分级标准, 在本研究中, 我们将从根桩上生长出 来的根定义为一级根, 生长在一级根上的子级根定 义为二级根, 二级根上生长的子级根定义为三级根, 以此类推。将每一级根认定为由下一级根分割的多 个连结, 记录每段连结的根系等级和连结顺序, 记 录方式图解见Ozier-Lafontaine和Sillon (1999), 测定 每条根系的基径以及每段根系连结的长度(连结长 度), 每条根的各段连接之和即为这条根的根系长 度, 每级根内各条根总数即为这一级根的根系数 量。测量至基径 $2 \mathrm{~mm}$ 的根系(因为 $2 \mathrm{~mm}$ 以下的根系 在挖掘过程中极难保留, 且 $2 \mathrm{~mm}$ 以上的根系足以 描述一个成熟林木的完整根系构型和分析其根系功 能, 因此本研究所提到的根系系统指的是基径 $2 \mathrm{~mm}$ 以上的完整根系系统)。根据此原始数据, 进行 各级根系形态结构分析并计算拓扑指标(Fitter \&

表1 毛白杨纯林与毛白杨刺槐混交林林地基本信息表

Table 1 General information of sample plots in Populus tomentosa stand and mixtures with P. tomentosa and Robinia pseudoacacia

\begin{tabular}{|c|c|c|c|c|c|c|}
\hline $\begin{array}{l}\text { 林型 } \\
\text { Stand type }\end{array}$ & $\begin{array}{l}\text { 树种 } \\
\text { Tree species }\end{array}$ & $\begin{array}{c}\text { 平均胸径 } \\
\text { Average } \\
D B H(\mathrm{~cm})\end{array}$ & $\begin{array}{c}\text { 平均树高 } \\
\text { Average tree } \\
\text { height }(\mathrm{m})\end{array}$ & $\begin{array}{c}\text { 株行距 } \\
\text { Plant \& row } \\
\text { spacing }(\mathrm{m})\end{array}$ & $\begin{array}{c}\text { 平均冠幅 } \\
\text { Average } \\
\text { crown width (m) }\end{array}$ & $\begin{array}{l}\text { 间伐措施 } \\
\text { Thinning treatment }\end{array}$ \\
\hline $\begin{array}{l}\text { 毛白杨纯林 } \\
\text { Populus tomentosa stand }\end{array}$ & $\begin{array}{l}\text { 毛白杨 } \\
\text { P. tomentosa }\end{array}$ & 36.1 & 35.5 & $6 \times 6$ & 7.0 & $\begin{array}{l}\text { 造林后第十年间伐除去刺槐, 以前的混交林 } \\
\text { 成为现有纯林 } \\
\text { All R. pseudoacacia trees were removed from } \\
\text { mixed-species stand after } 10 \text { years of planting, } \\
\text { resulting in P. tomentosa pure stand }\end{array}$ \\
\hline $\begin{array}{l}\text { 毛白杨×刺槐混交林 } \\
\text { Mixtures with P. tomentosa } \\
\text { and Robinia pseudoacacia }\end{array}$ & $\begin{array}{l}\text { 毛白杨 } \\
\text { P. tomentosa }\end{array}$ & 37.0 & 26.4 & $3 \times 6$ & 6.6 & 无 None \\
\hline $\begin{array}{l}\text { 毛白杨×刺槐混交林 } \\
\text { Mixtures with P. tomentosa } \\
\text { and R. pseudoacacia }\end{array}$ & $\begin{array}{l}\text { 刺槐 } \\
R . p s e u d o a c a c i a\end{array}$ & 25.1 & 21.1 & $3 \times 6$ & 8.6 & 无 None \\
\hline
\end{tabular}


Stickland, 1991; Oppelt et al., 2001)。

所有的数据整理在Excel 2016中完成。单因素方 差分析和协方差分析在SPSS 13.0里完成, 采用单因 素方差分析方法分析不同根系等级之间形态指标的 差异, 采用协方差分析方法对线性拟合方程的斜率 做差异显著性检验。在Origin 2017中进行线性拟合、 指数拟合和单峰函数拟合 $\left(\right.$ 其方程、 $p$ 值以及 $R^{2}$ 与在 $\mathrm{R}$ 语言中得出的结果一致), 并制作箱线图、拟合图和 组图。

\section{2 结果}

\section{1 根系最大生长空间}

纯林毛白杨、混交林毛白杨、混交林刺槐根系 系统水平延伸范围(距根桩直线距离)最远分别为 7.6、13.5和 $8.5 \mathrm{~m}$, 垂直扎根距离(距地面直线距离) 最深分别为 $5.7 、 6.4$ 和 $5.9 \mathrm{~m}$ (表2)。在水平方向上, 所 追踪的根系均未达到最终状态, 对于一些向下生长 的根系, 其极有可能再重新向水平方向生长, 因此 根系的水平生长最远空间不能确定。还有一部分根 是与同种另一株树的根系连生, 在纯林和混交林中 均发现这种现象。在混交林中未发现不同树种之间 的根系连生, 虽然有严重的挤压、扭曲和变形使其 外形极像连生, 但它们仍独立生长, 韧皮部无黏合
的痕迹。而在垂直范围内，大部分根系都能追踪到 头, 末端直径0.02-0.76 mm。

从所挖掘根系的生长状态来看, 所研究的根系 系统属于二态型根系(图1)。受到降水入渗深度的影 响，大部分的根系集中在1.2-1.5 m, 受到地下水位 的影响, 有一部分根会向下扎根直至地下水位, 但 这一部分根非常少, 且会以较细直径和较低直径变 化率向下延伸。

\section{2 根系系统中拓扑指标计算}

3 个根系系统的修正拓扑指数 $q_{a} 、 q_{b}$ 均接近于 0 $\left(q_{a}=0.02 、 0.03 、 0.02, q_{b}=-0.0002 、 0.0040 、-0.0060\right)$, 以及拓扑指数 $T I$ 均接近于 $0.5(T I=0.50 、 0.56 、 0.50)$ (表3)。说明3个根系系统均接近于叉状分枝(其中鱼 尾形分支 $q_{a}=q_{b}=1, T I=1$; 叉状形分支 $q_{a}=q_{b}=0$, $T I=0.5)$ 。

\section{3 各级根系基部直径、长度、数量的形态特征}

基部直径和根系长度随着根系等级增加而减小, 最后趋于平稳(图2)。3 个根系系统的基部直径都是 一级根最大, 3 个根系系统的一级根平均基径分别为 $10.60 、 10.54$ 和 $7.38 \mathrm{~cm}$, 分别是后几级根的 5.81-21.21、8.38-26.35和5.79-36.92倍。且一、二级 根与后几级根都差异极显著 $(p<0.0001)$, 而从三级 根开始其根系基部直径差异不显著 $(p>0.05)$ 。 3 个

表2 毛白杨及刺槐 3 个根系系统的被追踪根系终点状态描述

Table 2 Description of end states of the tracked roots in three root systems

\begin{tabular}{|c|c|c|c|c|c|c|c|}
\hline & \multirow[b]{2}{*}{$\begin{array}{c}\text { 追踪根系编号 } \\
\text { Code of the } \\
\text { tracked roots }\end{array}$} & \multicolumn{2}{|c|}{$\begin{array}{c}\text { 纯林毛白杨 } \\
\text { Populus tomentosa in pure stand }\end{array}$} & \multicolumn{2}{|c|}{$\begin{array}{c}\text { 混交林毛白杨 } \\
\text { P. tomentosa in mixed stand }\end{array}$} & \multicolumn{2}{|c|}{$\begin{array}{c}\text { 混交林刺槐 } \\
\text { Robinia pseudoacacia in mixed stand }\end{array}$} \\
\hline & & $\begin{array}{l}\text { 距离/深度 } \\
\text { Distance/ } \\
\text { depth }(\mathrm{m})\end{array}$ & $\begin{array}{l}\text { 终点状态 } \\
\text { End state }\end{array}$ & $\begin{array}{l}\text { 距离/深度 } \\
\text { Distance/ } \\
\text { depth }(\mathrm{m})\end{array}$ & $\begin{array}{l}\text { 终点状态 } \\
\text { End state }\end{array}$ & $\begin{array}{l}\text { 距离/深度 } \\
\text { Distance/ } \\
\text { depth (m) }\end{array}$ & $\begin{array}{l}\text { 终点状态 } \\
\text { End state }\end{array}$ \\
\hline \multirow{5}{*}{$\begin{array}{l}\text { 水平根 } \\
\text { Lateral } \\
\text { root }\end{array}$} & 1 & 3.3 & $\begin{array}{l}\text { 萌生幼树 } \\
\text { Sapling from root sprouting }\end{array}$ & 8.6 & $\begin{array}{l}\text { 与邻株连生 } \\
\text { Root grafted with neighbor tree }\end{array}$ & 6.5 & $\begin{array}{l}\text { 垂直向下生长 } \\
\text { Vertically downward growth }\end{array}$ \\
\hline & 2 & 5.1 & $\begin{array}{l}\text { 与邻株连生 } \\
\text { Root grafted with neighbor tree }\end{array}$ & 11.1 & 分叉 Branching & 8.2 & 分叉 Branching \\
\hline & 3 & 6.1 & $\begin{array}{l}\text { 垂直向下生长 } \\
\text { Vertically downward growth }\end{array}$ & 13.5 & $\begin{array}{l}\text { 垂直向下生长 } \\
\text { Vertically downward growth }\end{array}$ & 8.5 & $\begin{array}{l}\text { 垂直向下生长 } \\
\text { Vertically downward growth }\end{array}$ \\
\hline & 4 & 6.4 & $\begin{array}{l}\text { 垂直向下生长 } \\
\text { Vertically downward growth }\end{array}$ & & & & \\
\hline & 5 & 7.6 & $\begin{array}{l}\text { 垂直向下生长 } \\
\text { Vertically downward growth }\end{array}$ & & & & \\
\hline \multirow[t]{4}{*}{$\begin{array}{l}\text { 垂直根 } \\
\text { Vertical } \\
\text { root }\end{array}$} & 1 & 3.1 & $\begin{array}{l}\text { 到头, 末端直径 } 0.3 \mathrm{~mm} \\
\text { To the end, root tip } \\
\text { diameter } 0.3 \mathrm{~mm}\end{array}$ & 4.0 & $\begin{array}{l}\text { 到头, 末端长瘤 } \\
\text { To the end, with tumor }\end{array}$ & 2.7 & $\begin{array}{l}\text { 坚直向上生长 } \\
\text { Vertically upward growth }\end{array}$ \\
\hline & 2 & 4.3 & $\begin{array}{l}\text { 到头, 末端直径 } 0.3 \mathrm{~mm} \\
\text { To the end, root tip } \\
\text { diameter } 0.3 \mathrm{~mm}\end{array}$ & 4.3 & $\begin{array}{l}\text { 到头, 末端直径 } 0.5 \mathrm{~mm} \\
\text { To the end, root tip } \\
\text { diameter } 0.5 \mathrm{~mm}\end{array}$ & 3.7 & $\begin{array}{l}\text { 到头, 末端直径 } 0.3 \mathrm{~mm} \\
\text { To the end, root tip } \\
\text { diameter } 0.3 \mathrm{~mm}\end{array}$ \\
\hline & 3 & 5.7 & $\begin{array}{l}\text { 到头, 末端直径 } 0.25 \mathrm{~mm} \\
\text { To the end, root tip } \\
\text { diameter } 0.25 \mathrm{~mm}\end{array}$ & 4.3 & $\begin{array}{l}\text { 到头, 末端直径 } 0.76 \mathrm{~mm} \\
\text { To the end, root tip } \\
\text { diameter } 0.76 \mathrm{~mm}\end{array}$ & 5.9 & $\begin{array}{l}\text { 到头, 末端直径 } 0.02 \mathrm{~mm} \\
\text { To the end, root tip } \\
\text { diameter } 0.02 \mathrm{~mm}\end{array}$ \\
\hline & 4 & & & 6.4 & 向西转弯 Bend to the west & & \\
\hline
\end{tabular}



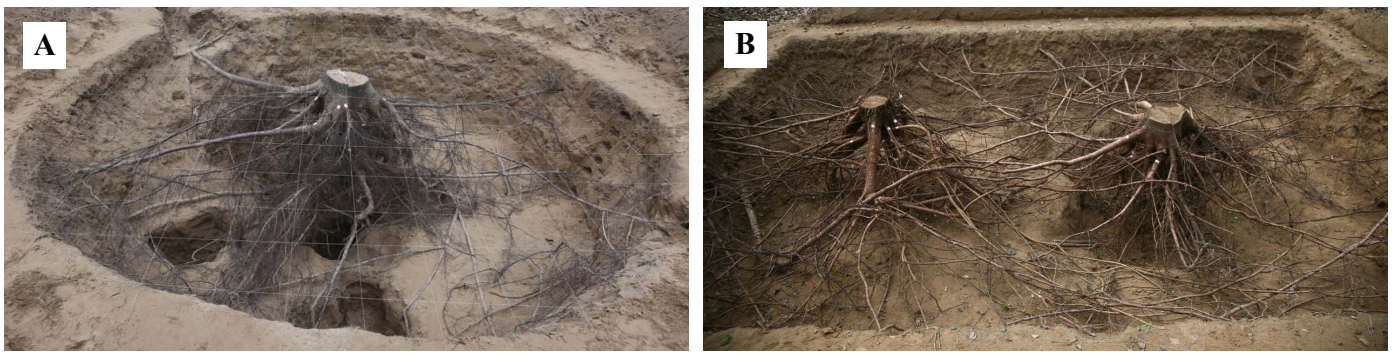

图1 纯林毛白杨 $(\mathbf{A})$ 、混交林毛白杨和刺槐 $(\mathbf{B})$ 根系系统实拍图(镜头位置为东边)。

Fig. 1 Photos of root systems of Populus tomentosa in pure stand (A), P. tomentosa and Robinia pseudoacacia in mixed stand (B) (Lens position to the east).

表3 毛白杨及刺槐 3 个根系系统拓扑指标参数

Table 3 The values of the topological indices for three root systems

\begin{tabular}{lcrrrrr}
\hline 根系系统 Root system & $a(A)$ & $P_{e}$ & $V_{0}(M)$ & $b$ & $q_{a}$ & $q_{b}$ \\
\hline 纯林毛白杨 Populus tomentosa in pure forest & 29 & 8367 & 793 & 10.551 & 0.02 & -0.0002 \\
混交林毛白杨 P. tomentosa in mixed forest & 61 & 21322 & 1456 & 14.644 & 0.50 \\
混交林刺槐 Robinia pseudoacacia in mixed forest & 21 & 3990 & 465 & 8.581 & 0.02 & -0.0060 \\
\hline
\end{tabular}

$a(A)$, 最长通道根基到根终端连结数量; $P_{e}$, 所有通道根基到根终端通道的连结总数; $V_{0}(\mathrm{M})$, 外部连结总和; $b$, 根系平均拓扑长度 $\mathrm{b}=P e / V_{0} ; q_{a}$ $=\frac{a-1-\ln V_{0} / \ln 2}{V_{0}-1-\ln V_{0} / \ln 2} ; q_{b}=\frac{b-1-\ln V_{0} / \ln 2}{\frac{V_{0}+1}{2}-V_{0}^{-1}-\ln V_{0} / \ln 2} ; T I=\lg A / \lg M($ Oppelt et al., 2001).

$a(A)$, number of links in the longest unique path from the base link to an exterior link; $P_{e}$, sum of numbers of links in all possible unique paths from the base link to all exterior links; $V_{0}(M)$, number of exterior links; $b$, average topological length $b=P e / V_{0} ; q_{a}=\frac{a-1-\ln V_{0} / \ln 2}{V_{0}-1-\ln V_{0} / \ln 2} ; q_{b}=\frac{b-1-\ln V_{0} / \ln 2}{\frac{V_{0}+1}{2}-V_{0}^{-1}-\ln V_{0} / \ln 2} ; T I=$

$\lg A / \lg M$ (Oppelt et al., 2001).
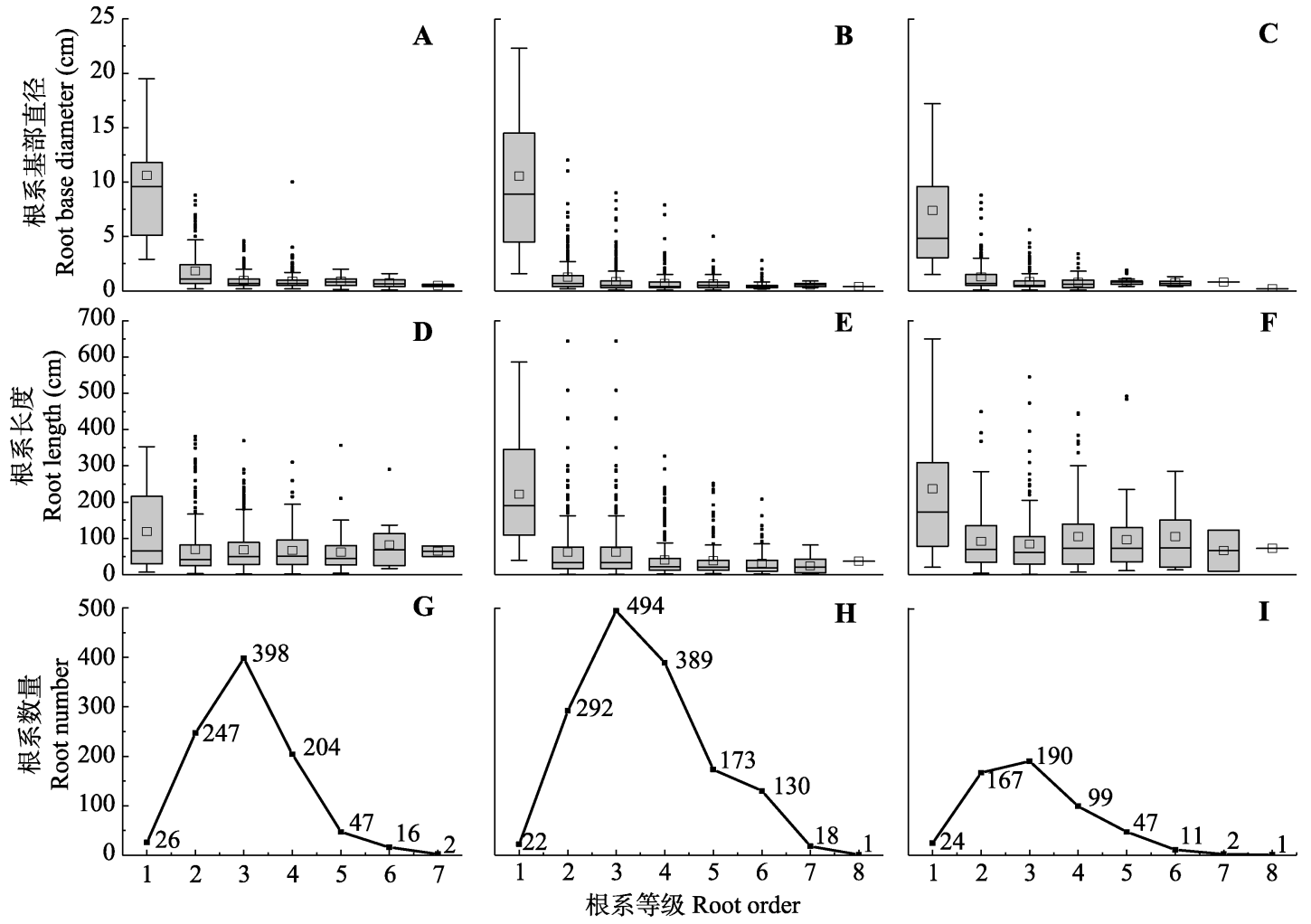

图2 纯林毛白杨 $(\mathbf{A} 、 \mathbf{D} 、 \mathbf{G}) 、$ 混交林毛白杨 $(\mathbf{B} 、 \mathbf{E} 、 \mathbf{H})$ 和刺槐 $(\mathbf{C} 、 \mathbf{F 、 I}$ 根系系统不同等级根系基部直径、长度、数量的分 布格局图。箱线图上的虚点代表异常点。箱线图上的正方形代表平均值。

Fig. 2 Variations of basal diameter, length, and number of roots for different order roots in root systems of Populus tomentosa in pure forest (A, D, G), P. tomentosa in mixed forest (B, E, H), and Robinia pseudoacacia in mixed forest $(\mathbf{C}, \mathbf{F}, \mathbf{I})$. The individual points on the box plots represent outliers, the squares on the box plots represent the average. 
根系系统的根系长度都是一级根最长, 且一级根都 显著高于后几级根 $(p<0.05)$, 三个根系系统的一级 根平均长度分别是 $118.90 、 221.71$ 和 $236.81 \mathrm{~cm}$, 分别 是后几根的1.45-1.95、3.55-9.11和2.26-3.56倍。从 二级根开始各级根系长度差异不显著 $(p>0.05)$ 。根 系数量随着根系等级增加先增加后降低, 在三级根 上达到最高, 分别为纯林毛白杨398条、混交林毛白 杨494条、混交林刺槐190条。

为了能更加清楚地表达根系等级与根系基径、 长度、数量的关系, 我们将各形态指标与根系等级 之间进行了拟合(表4)。各形态指标与根系等级之间 都有着极显著的相关性 $(p<0.01)$ 。根系的基径、长 度与根系等级之间都可以用指数函数拟合, 基径与 根系等级的拟合优度较高 $\left(R^{2}=0.48 、 0.41 、 0.37\right)$, 但 长度与根系等级的却很低 $\left(R^{2}=0.01 、 0.12 、 0.08\right)$, 根 系数量与根系等级之间用Origin软件单峰拟合的拟 合优度很高 $\left(R^{2}=0.99 、 0.91 、 0.94\right)$ 。

\section{4 各级根系从根基到根尖各段连结长度变化探究}

从图3可以看出, 前三级根根系从根基到根尖, 各段连结长度的变化不大。为了能够更加直观地分 析这种趋势, 我们在每个根系系统的前三级根中各 挑选5-8 个连结数量靠前的根, 将连结顺序(从根基 到根尖)与累积连结长度做线性拟合, 发现平均决 定系数 $R^{2}$ 为 $0.87, p$ 值均小于 0.05 (数据未展示)。

\section{5 各级根系作为母级根与其子级根关于基部直 径的拓扑关系}

母级根基部直径与其子级根基部直径平均值存 在正相关的关系, 且相关关系极显著 $(p<0.0001)$ (图4)。以一级根为母级根的子级根基部直径与母级
根基部直径的线形方程决定系数均大于 0.5 , 而以 二、三级根为母级根的决定系数均大于 0.7 , 在以二、 三级根为母级根的母级根与子级根基部直径相关关 系上比以一级根为母级根有着更好的线形关系。以 一级根为母级根的线形回归方程斜率 $(0.13 、 0.14$ 、

$0.17)$ 明显要低于以二级根 $(0.35 、 0.31 、 0.37)$ 、三级 根 $(0.39 、 0.25 、 0.40)$ 为母级根 $(p<0.01)$, 而以二、 三级根为母级根之间的斜率相差不大 $(p>0.05)$ 。

\section{6 各级根系基部直径与根系长度的相关关系}

根系基部直径与根系长度均呈现正相关关系, 除刺槐一级根 $(p>0.05)$ 以外, 其他线形方程的相关 关系显著 $(p<0.05 、 p<0.01 、 p<0.001 、 p<$ 0.0001 )(图5)。刺槐一级根的线性方程拟合优度较低 $\left(R^{2}=0.09\right)$, 其他的基部直径与根系长度的线性方 程决定系数 $R^{2}$ 都在 0.3 以上。随着根系等级的增高, 三个根系系统的根系基部直径与根系长度的线形回 归方程斜率均呈现增加的趋势, 其中纯林毛白杨的 线性方程斜率从 8.04 增长至93.93, 混交林毛白杨从 11.53 增长至 48.05 , 混交林刺槐从 11.80 增长至 129.31 。

\section{3 讨论}

\section{1 砂壤土上成熟林木生长空间}

植物根系分布深度受树种(Fan et al., 2017), 降 水(Schenk \& Jackson, 2002a, 2005), 土壤厚度和土 壤质地、地形(Schenk \& Jackson, 2005; Xu \& Li, 2008; Fan et al., 2017), 地下水位(Fan et al., 2017; Di et al., 2018)等影响。本研究中根系的扎根深度最远 达到 $6.4 \mathrm{~m}$ (研究地的地下水位 $6 \mathrm{~m}$ 左右), 而根系系

表4 毛白杨与刺槐根系基径、长度、数量与根系等级的拟合结果

Table 4 Results of fitting regression models of Populus tomentosa and Robinia pseudoacacia-regressing root diameter, length, and numbers (y) on root orders $(x)$, respectively

\begin{tabular}{|c|c|c|c|c|}
\hline $\begin{array}{l}\text { 根形态指标 } \\
\text { Root morphological } \\
\text { characteristics }\end{array}$ & 根系系统 Root system & 方程 Regression model & $\begin{array}{c}\text { 拟合优度 } \\
R^{2}\end{array}$ & $\begin{array}{c}\text { 样本量 } \\
n\end{array}$ \\
\hline \multirow{3}{*}{$\begin{array}{l}\text { 基径 } \\
\text { Basal diameter }\end{array}$} & 纯林毛白杨 P. tomentosa in pure forest & $y=0.86381+99.24471 \exp (-2.32112 x)$ & 0.4840 & 940 \\
\hline & 混交林毛白杨 P. tomentosa in mixed forest & $y=0.70036+157.41951 \exp (-2.77342 x)$ & 0.4149 & 1527 \\
\hline & 混交林刺槐 R. pseudoacacia in mixed forest & $y=0.80671+91.76619 \exp (-2.63586 x)$ & 0.3747 & 541 \\
\hline \multirow{3}{*}{$\begin{array}{l}\text { 长度 } \\
\text { Length }\end{array}$} & 纯林毛白杨 P. tomentosa in pure forest & $y=67.63168+1098.3759 \exp (-3.32236 x)$ & 0.0146 & 940 \\
\hline & 混交林毛白杨 P. tomentosa in mixed forest & $y=39.71891+1313.84526 \exp (-1.98679 x)$ & 0.1172 & 1527 \\
\hline & 混交林刺槐 R. pseudoacacia in mixed forest & $y=92.10741+477664.65953 \exp (-8.10278 x)$ & 0.0795 & 541 \\
\hline \multirow{3}{*}{$\begin{array}{l}\text { 数量 } \\
\text { Numbers }\end{array}$} & 纯林毛白杨 P. tomentosa in pure forest & $y=5.97738+396.37817 \exp \left(-0.5((x-2.93757) / 0.90608)^{2}\right)$ & 0.9895 & 7 \\
\hline & 混交林毛白杨 P. tomentosa in mixed forest & $y=22.41349+474.80796 \exp \left(-0.5((x-3.28655) / 1.13184)^{2}\right)$ & 0.9127 & 8 \\
\hline & 混交林刺槐 $R$. pseudoacacia in mixed forest & $y=5.83356+196.21954 \exp \left(-0.5((x-2.79311) / 1.01443)^{2}\right)$ & 0.9367 & 8 \\
\hline
\end{tabular}

www.plant-ecology.com 

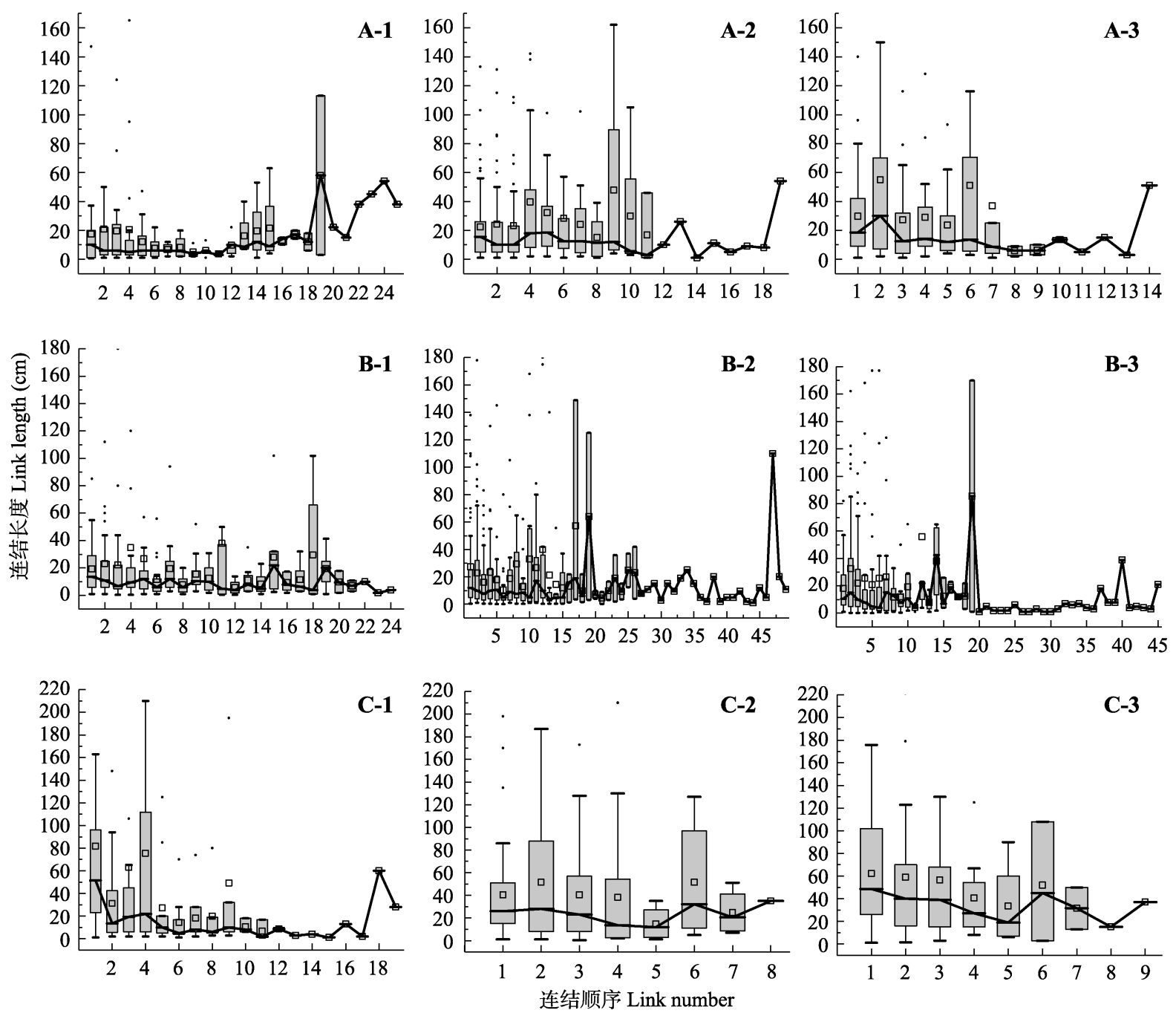

图3 纯林毛白杨 $(\mathbf{A})$ 、混交林毛白杨 $(\mathbf{B})$ 和刺槐 $(\mathbf{C})$ 根系系统根基到根尖各段连结长度变化趋势图。 1 , 一级根。 2 , 二级根。3, 三级根。箱线图上的虚点代表异常点。箱线图上的正方形代表平均值。箱线图上的线条为中位数的连线。

Fig. 3 Link length variations along link sequence from the root base to the root tip in root systems of Populus tomentosa in pure forest (A), P. tomentosa in mixed forest (B), and Robinia pseudoacacia in mixed forest (C). 1-3 refer to root categories from first order to third order. The individual points on the box plots represent outliers, the squares on the box plots represent the average, and the lines connected the box plots.

统主体分布在 $1.5 \mathrm{~m}$ 以上的土壤层。造成这种现象的 原因可能是根系为适应季节性的降水，提高了湿润 季的吸水效率，使根系在浅土层集中分布(De Deurwaerder et al., 2018), 同时湿润季节较深的降雨入 渗会诱导深土层根系的增殖, 促进形成相对较深的 根系分布，在这个过程中，砂壤土的质地还可以调 节水的向下渗透和向上毛细提升, 共同塑造根系的 深层扎根(Fan et al., 2017), 形成的深层根系可以利 用地下水来缓解旱季的缺水(Di et al., 2018)。

由于追踪的水平根生长到一定范围向下生长, 因此根系的水平空间范围可能会被低估，但多次挖 掘出来的同种树之间的根系连生可能扩大了根系的
资源探索空间。在季节性水资源有限的环境中，例 如季节性干旱或持续洪水发生的地方，许多树种可 能在浅层土壤中具有发育良好的侧根(Shafroth et al., 2000; Schenk \& Jackson, 2002b)。试验地属暖温 带半干旱季风区域大陆性气候，具有显著的季节变 化，降水集中在7-8月，其他季节少雨。本研究的侧 根平均水平范围最远达到 $13.5 \mathrm{~m}$ (冠半径的4.09倍), 表明在砂壤土上成熟林木的根系可以在少雨季节拦 截珍贵的降雨, 通过探索较大的土壤区域吸收和运 输长距离的土壤水分和养分(Read, 1991; Bakker et al., 2006)。因此, 开发这种广泛扩展的根系可能是 砂壤土上成熟林木克服季节性干旱并同时吸收土壤 


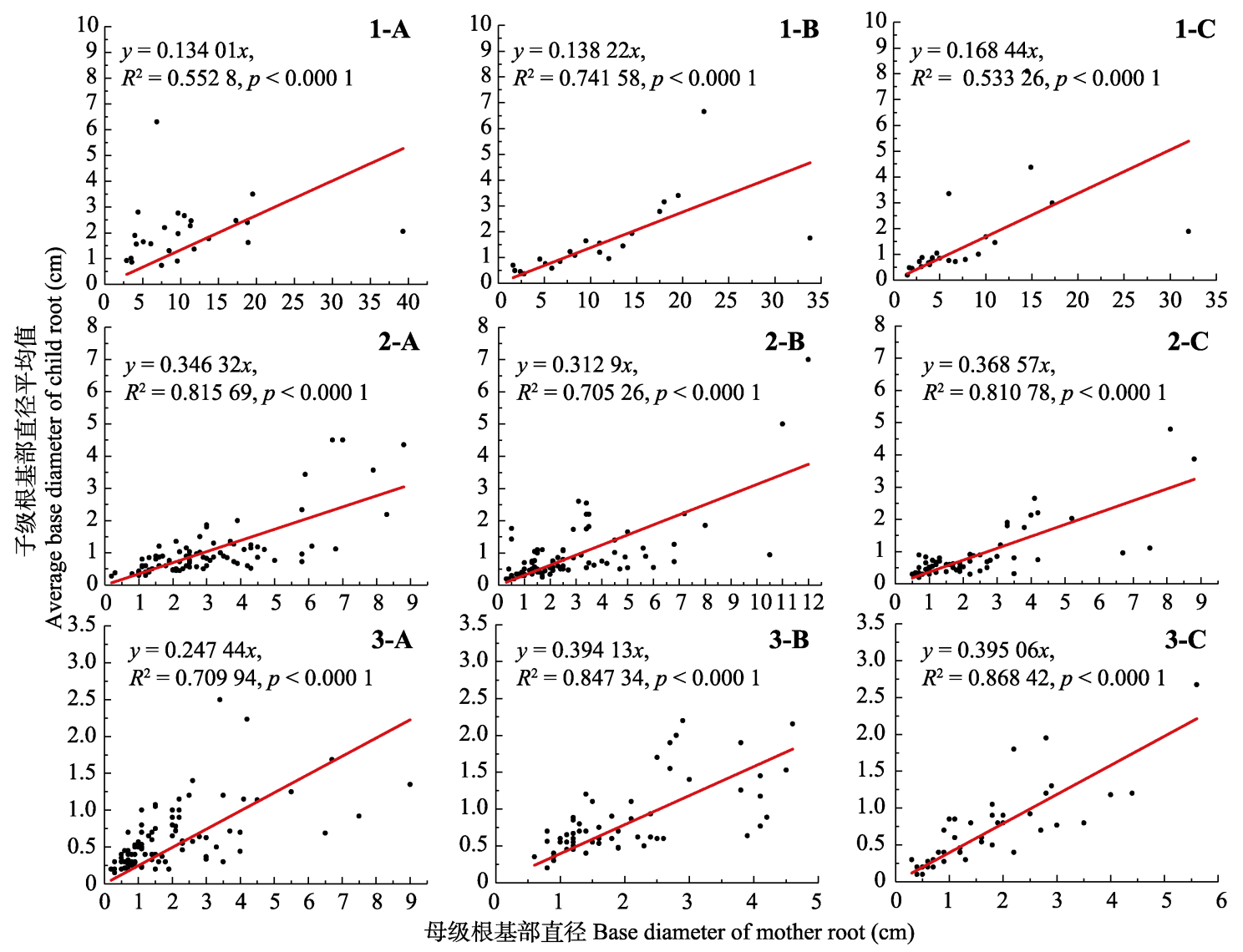

图4 母级根基部直径与子级根基部直径的相关关系图。1 , 以一级根为母级根。2 , 以二级根为母级根。 3 , 以三级根为母级 根。A, 纯林毛白杨根系系统。 $\mathbf{B}$, 混交林毛白杨根系系统。 $\mathbf{C}$, 混交林刺槐根系系统。

Fig. 4 Basal diameter relationships between the mother roots and the child roots. 1, refer to mother-root categories from first order. 2, refer to mother-root categories from second order. 3, refer to mother-root categories from third order. A, root system of Populus tomentosa in pure forest. $\mathbf{B}$, root system of $P$. tomentosa in mixed forest. $\mathbf{C}$, root system of Robinia pseudoacacia in mixed forest.

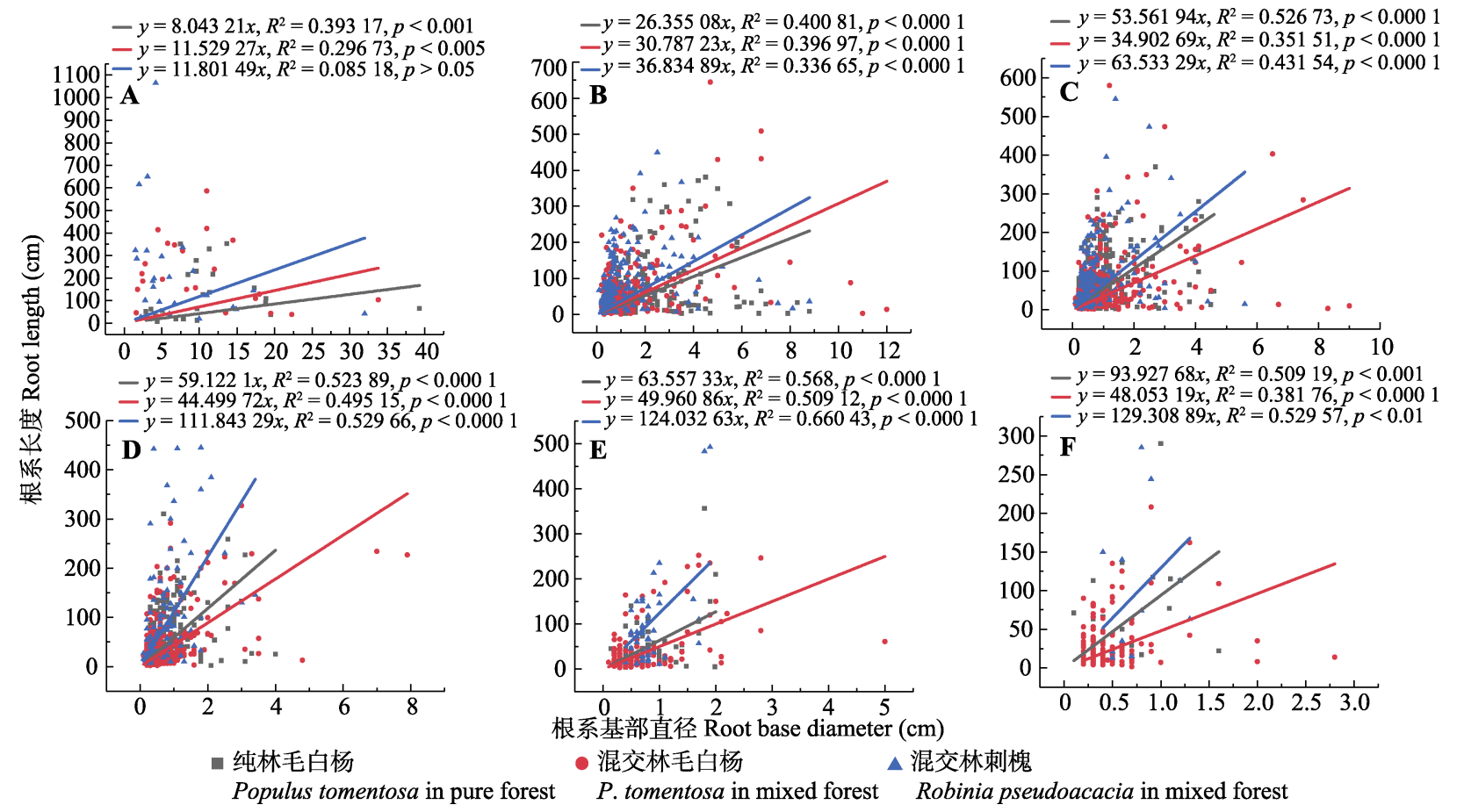

图5 不同等级根系的基部直径与根系长度的关系图。A-F，一到六级根。

Fig. 5 Relationships between basal diameter and root length for different order roots. A-F refer to root categories from first order to sixth order.

www.plant-ecology.com 
资源的一种适应性策略。

深根和广泛侧根的同时发育也表明砂壤土上的 成熟林木具有二态型根系(Pate et al., 1995; Dawson \& Pate, 1996; Fan et al., 2017)以适应这种季节性水 分限制环境。

\section{2 各级根系形态特征}

本研究是以Rose (1983)提出的发育命名系统为 根系分级标准, 将从根桩上生长出来的根作为一级 根, 从一级根上长出来的根定义为二级根, 以此类 推, 上下级根是连续承载的关系, 最外围的根存在 各级根, 按照这种分级方法, 根系数量随根序的变 化趋势是存在多种可能的。而常见的研究细根的分 级系统(Pregitzer et al., 2002)是将根系系统最外围 的根尖作为一级根, 两个一级根相会形成二级根, 以此类推, 上下级根是融合的关系, 最外围的根只 有一级根, 按照细根的分级方法, 根系数量随根序 的增加只能单调递减。所以两种分级方法的逻辑是 完全不同的, 得出来的关于根系数量的结果肯定是 不一样的。在发育分级系统下, Ozier-Lafontaine等 (1999)在对3.5年生的木本植物Gliricidia sepium的研 究中发现随着根系等级的增加, 根系数量先增后减, 根系数量在二级根上达到最高, 但本研究的根系数 量是在三级根上达到峰值, 这与一级根的定义有关, 本研究将根桩上生长的主根定义为一级根, 而 Ozier-Lafontaine等(1999)将主根上生长的侧根定义 为一级根, 因此本研究比 Ozier-Lafontaine等(1999) 的研究要多一级的根系。

研究发现 3 个根系系统根系基部直径随着根系 等级的增加逐渐降低并趋于平稳, 根系基部直径与 根系等级呈现指数关系。Ozier-Lafontaine等(1999) 也发现根系等级分别与平均基径和平均长度有较高 的拟合优度。Pregitzer等(1997)用发育分级方法在 Acer saccharum和美国白蜡(Fraxinus americana)的 幼苗上也发现随着根系等级的增加根系直径逐渐降 低, 并且一级根的直径明显高于后几级根。根尖直 径与根系等级也是一种负相关的关系(Pagès et al., 2013), 因此根系基径与根尖直径可能也存在某种 关系, 但尚未有实验验证。虽然本研究的分级方法 与细根分级方法不同, 但我们发现细根的根序和直 径的关系也可以用指数函数表示, 根序与根系直径 之间是正相关关系(常文静和郭大立, 2008)。结合本 研究对直径 $2 \mathrm{~mm}$ 以上的根系系统的直径研究和以
往细根的研究, 可以发现沿着根桩向外围根尖扩散 方向, 整个系统的根系直径大致呈现逐渐递减的趋 势。

根系长度与根系等级存在负相关关系, 其线性 方程拟合优度较低, 这是由于各级根系的长度存在 很大变异性所导致的。根系长度由根基部直径与根 直径纵向变化模式共同决定(吴茜, 2016), 还有在一 些外部因素影响根系长度, 比如土壤温度(Tardieu \& Pellerin, 1991; Nagel et al., 2009)、土壤穿透力 (Bécel et al., 2012)、局部养分可利用性以及养分斑 块的存在(Yu et al., 2015)等等，同时，通过挖掘法 在野外获取的根系经常容易断根, 这些实验误差也 会对实验结果造成一定的影响。根系连结长度作为 拓扑指标中一个重要指标, 反映着根系探索资源和 拓展空间的能力(Fitter \& Stickland, 1991; Berntson, 1994)。根系连结长度沿根系生长方向的变化可以用 来表征根系延伸策略，根系延伸策略反映了立地的 土壤养分条件以及植物的受养特征(赵艳云等, 2015)。本研究就此做了相关研究: 沿着根系的生长 方向, 三个根系系统的连结长度变化不大, 与赵艳 云等 (2015) 对杜柳 (Periploca sepium) 和酸束 (Ziziphus jujuba) 的研究得出的规律相同。说明: 1) 根系在延伸过程中遇到的土壤条件异质性较低；2) 子级根在母级根上的均匀分布, 让整个根系系统成 为一个均匀的系统, 使吸收根能够更充分地利用土 壤养分和水资源。同时，连结顺序(从根基到根尖) 与累积连结长度呈现线性关系, 这也为我们预测根 系模型奠定了理论基础。

\section{3 各级根系功能分析}

子级根与母级根之间在直径上存在显著的相关 性, 并且这种关系适用于广泛的物种(Lecompte et al., 2005; Bécel et al., 2012; Wu et al., 2016)。早期的 这些研究对多种双子叶和单子叶植物的母级根直径 和子级根直径的相关关系进行了量化分析, 并对比 了不同物种(Pagès, 2014)、不同处理(Bécel et al., 2012; Wu et al., 2016)间这种相关关系的差异(即斜 率比较), 但没有将不同根系等级间这种关系考虑 进去。本研究发现成熟林木的母级根直径与子级根 直径同样存在着显著正相关关系, 拟合了无截距的 线性方程, 其斜率就是子级根与母级根直径的比率, Bécel等(2012)曾用子级根与母级根直径的比率变化 来描述根分支模式, 比率高说明子级根直径增加超 
过母级根，根分枝模式倾向于二分型，而不是“鲱鱼 骨”形式(严小龙等, 2000), 联系到本研究, 斜率越 高说明根系在发育时越倾向于优先发育子级根, 斜 率越低则越优先发育母级根。本研究发现一级根作 为母级根与其子级根的基径拟合线性方程斜率相对 于二、三级根最低, 即一级根会倾向于优先发育自 身直径, 且在发育命名系统下, 一级根在基径和长 度上明显高于后几级根, 主根 (即本研究中的一级 根)的尺寸(直径和长度)是影响根系系统针固功能的 重要因素(Yang et al., 2018), 说明一级根在整个根 系系统中主要承担着支撑和针固的作用; 二级根之 后的根系相对一级根则会优先发育子级根直径, 通 过不断增强子级根以达到高效占领土壤资源和空间 的目的。通过拓扑指数我们可以发现本研究的 3 个根 系系统的整体构型都是叉状分支 (二分型), 因此如 果研究材料的根系是“鲱鱼骨”形式(严小龙等, 2000), 那么所得出的规律可能会有所不同, 更多的 规律还需要大量的试验去证明。

根系直径与长度的正相关关系, 在垂枝桦 (Betula pendula)、欧洲云杉(Picea abies)和欧洲赤松 (Pinus sylvestris)三个树种的成熟林木中已被发现 (Kalliokoski et al., 2010); 吴茜(2016)对单子叶植物 玉米(Zea mays)的研究中也发现了这种规律。但是尚 未有研究将根系直径与长度的这种关系延伸到不同 根系等级上, 尤其是成熟林木的完整根系系统。前 面已经提到, 根系长度反映着根系探索资源和拓展 空间的能力 (Fitter \& Stickland, 1991; Berntson, 1994)。在本研究中从一级根到六级根, 根系长度与 基径的线性方程斜率(无截距)逐渐增高, 其斜率就 是根系长度与根系直径的比率, 斜率的增高说明长 度增加比直径增加得快, 这也意味着越高级别的根 系, 可能会更加倾向于通过增长根系长度来达到探 索资源、拓展空间的目的。当然, 与母级根和子级 根关系的情况一样, 如果研究材料是“鲱鱼骨”形式 的根系, 那么研究结果可能存在差异, 需要更多试 验去证明。

综合以上，砂壤土上成熟林木的一级根会倾向 于积累自身生物量, 在整个根系系统中主要承担着 支撑和针固的作用, 二级根之后的根系相对一级根 则会倾向于优先发育其子级根以及自身根长生长, 在整个根系系统中主要承担着探索资源和扩展范围 的功能。

\section{4 结论}

本研究通过全挖法获取了砂壤土上毛白杨和刺 槐的 3 个成熟林木的完整根系系统, 基于根系发育 分级方法对 3 个根系系统进行形态和功能分析。研究 表明: 从根系系统的整体结构上看, 砂壤土上的成 熟林木通过垂直方向深根系和水平方向广泛分布以 及扩散型的叉状分支结构来拓展自己的分布空间从 而吸收更多的资源。从根系发育分级的角度来分析 根系的结构与功能, 成熟林木的一级根尺寸明显高 于后几级根, 并且一级根倾向于优先发育自身直径, 从而达到针固和支撑的作用; 而二级根之后的各级 根会倾向于优先发育子级根和增长根长, 以达到探 索资源、拓展空间的目的。

\section{参考文献}

Bakker MR, Augusto L, Achat DL (2006). Fine root distribution of trees and understory in mature stands of maritime pine (Pinus pinaster) on dry and humid sites. Plant and Soil, 286, 37-51.

Bécel C, Vercambre G, Pagès L (2012). Soil penetration resistance, a suitable soil property to account for variations in root elongation and branching. Plant and Soil, 353, 169-180.

Berntson GM (1994). Modelling root architecture: Are there tradeoffs between efficiency and potential of resource acquisition? New Phytologist, 127, 483-493.

Chang WJ, Guo DL (2008). Variation in root diameter among 45 common tree species in temperate, subtropical and tropical forests in China. Journal of Plant Ecology (Chinese Version), 32，1248-1257. [常文静, 郭大立 (2008). 中国温带、亚热带和热带森林 45 个常见树种细根直径变 异. 植物生态学报, 32, 1248-1257.]

Danjon F, Khuder H, Stokes A (2013). Deep phenotyping of coarse root architecture in $R$. pseudoacacia reveals that tree root system plasticity is confined within its architectural model. PLOS ONE, 8, e83548. DOI: 10.1371/journal.pone.0083548.

Danjon F, Reubens B (2008). Assessing and analyzing 3D architecture of woody root systems, a review of methods and applications in tree and soil stability, resource acquisition and allocation. Plant and Soil, 303, 1-34.

Danjon F, Barker DH, Drexhage M, Stokes A (2008). Using three-dimensional plant root architecture in models of shallow-slope stability. Annals of Botany, 101, 1281-1293.

Danjon F, Fourcaud T, Bert D (2005). Root architecture and wind-firmness of mature Pinus pinaster. New Phytologist, 168, 387-400.

Dawson TE, Pate JS (1996). Seasonal water uptake and movement

www.plant-ecology.com 
in root systems of Australian phraeatophytic plants of dimorphic root morphology: A stable isotope investigation. Oecologia, 107, 13-20.

De Deurwaerder H, Herve-Fernandez P, Stahl C, Burban B, Petronelli P, Hoffman B, Bonal D, Boeckx P, Verbeeck H (2018). Liana and tree below-ground water competitionEvidence for water resource partitioning during the dry season. Tree Physiology, 38, 1071-1083.

Di N, Liu Y, Mead DJ, Xie YQ, Jia LM, Xi BY (2018). Root-system characteristics of plantation-grown Populus tomentosa adapted to seasonal fluctuation in the groundwater table. Trees, 32, 137-149.

Fan Y, Miguez-Macho G, Jobbagy EG, Jackson RB, OteroCasal C (2017). Hydrologic regulation of plant rooting depth. Proceedings of the National Academy of Sciences of the United States of America, 114, 10572-10577.

Fitter AH (1986). The topology and geometry of plant root systems: Influence of watering rate on root system topology in trifolium pretense. Annals of Botany, 58, 91-101.

Fitter AH (1987). An architectural approach to the comparative ecology of plant root systems. New Phytologist, 106, 61-77.

Fitter AH, Stickland TR (1991). Architectural analysis of plant root systems: 2. Influence of nutrient supply on architecture in contrasting plant species. New Phytologist, 118, 383-389.

Gärtner H, Wagner B, Heinrich I, Denier C (2009). 3D-laser scanning: A new method to analyze coarse tree root systems. Forest Snow and Landscape Research, 82, 95-106.

Guo DL, Xia MX, Wei X, Chang WJ, Liu Y, Wang ZQ (2008). Anatomical traits associated with absorption and mycorrhizal colonization are linked to root branch order in twenty-three Chinese temperate tree species. New Phytologist, 180, 673-683.

Kalliokoski T, Sievänen R, Nygren P (2010). Tree roots as self-similar branching structures: Axis differentiation and segment tapering in coarse roots of three boreal forest tree species. Trees, 24, 219-236.

Lecompte F, Pages L, Ozier-Lafontaine H (2005). Patterns of variability in the diameter of lateral roots in the banana root system. New Phytologist, 167, 841-850.

Lynch J (1995). Root architecture and plant productivity. Plant physiology, 109, 7-13.

Nagel KA, Kastenholz B, Jahnke S, Van Dusschoten D, Aach T, Muhlich M, Truhn D, Scharr H, Terjung S, Walter A, Schurr U (2009). Temperature responses of roots: Impact on growth, root system architecture and implications for phenotyping. Functional Plant Biology, 36, 947-959.

Oppelt AL, Kurth W, Godbold DL (2001). Topology, scaling relations and Leonardo's rule in root systems from African tree species. Tree Physiology, 21, 117-128.

Osawa A, Zyryanova OA, Matsuura Y, Kajimoto T, Wein RW
(2010). Permafrost Ecosystems: Siberian Larch Forests. Springer, Dordrecht, the Netherlands.

Ozier-Lafontaine H, Sillon JF (1999). Fractal analysis of the root architecture of Gliricidia sepium for the spatial prediction of root branching, size and mass: Model development and evaluation in agroforestry. Plant and Soil, 209, 167-179.

Pagès L, Doussan C, Vercambre G (2000). An introduction on below-ground environment and resource acquisition, with special reference on trees. Simulation models should include plant structure and function. Annals of Forest Science, 57, 513-520.

Pagès L, Xie J, Serra V (2013). Potential and actual root growth variations in root systems: Modeling them with a two-step stochastic approach. Plant and Soil, 373, 723-735.

Pagès L (2014). Branching patterns of root systems: Quantitative analysis of the diversity among dicotyledonous species. Annals of Botany, 114, 591-598.

Pate JS, Jeschke WD, Aylward MJ (1995). Hydraulic architecture and xylem structure of the dimorphic root systems of South-West Australian species of Proteaceae. Journal of Experimental Botany, 46, 907-915.

Pregitzer KS, Kubiske ME, Yu CK, Hendrick RL (1997). Relationships among root branch order, carbon, and nitrogen in four temperate species. Oecologia, 111, 302-308.

Pregitzer KS, Deforest JL, Burton AJ, Allen MF, Hendrick RRL (2002). Fine root architecture of nine North American trees. Ecological Monographs, 72, 293-309.

Read DJ (1991). Mycorrhizas in ecosystems. Experientia, 47, 376-391.

Rose DA (1983). The description of the growth of root systems. Plant and Soil, 75, 405-415.

Salahuddin, Rewald B, Razaq M, Lixue Y, Li J, Khan F, Jie Z (2018). Root order-based traits of Manchurian walnut \& larch and their plasticity under interspecific competition. Scientific Reports, 8, 9815. DOI: 10.1038/s41598-01827832-0.

Schenk HJ, Jackson RB (2002a). The global biogeography of roots. Ecological Monographs, 72, 311-328.

Schenk HJ, Jackson RB (2002b). Rooting depths, lateral spreads, and below-ground/above-ground allometries of plants in water-limited ecosystems. Journal of Ecology, 90, 480-494.

Schenk HJ, Jackson RB (2005). Mapping the global distribution of deep roots in relation to climate and soil characteristics. Geoderma, 126, 129-140.

Shafroth PB, Stromberg JC, Patten DT (2000). Woody riparian vegetation response to different alluvial water table regimes. Western North American Naturalist, 60, 66-76.

Smit AL, Bengough AG, Engels C, Noordwijk M, Pellerin S, Geijn SC (2000). Root Methods. Springer, Berlin.

Strahler AN (1957). Quantitative analysis of watershed 
geomorphology. Eos Transactions American Geophysical Union, 38, 913-920.

Tardieu F, Pellerin S (1991). Influence of soil temperature during root appearance on the trajectory of nodal roots of field grown maize. Plant and Soil, 131, 207-214.

Valdes-Rodriguez OA, Sanchez-Sanchez O, Perez-Vazquez A, Caplan JS, Danjon F (2013). Jatropha curcas L. root structure and growth in diverse soils. The Scientific World Journal, 2013, 827295. DOI: 10.1155/2013/827295.

Vercambre G, Pages L, Doussan C, Habib R (2003). Architectural analysis and synthesis of the plum tree root system in an orchard using a quantitative modelling approach. Plant and Soil, 251, 1-11.

Vennetier M, Zanetti C, Meriaux P, Mary B (2015). Tree root architecture: New insights from a comprehensive study on dikes. Plant and Soil, 387, 81-101.

Wagner B, Gärtner H, Santini S, Ingensand H (2011). Crosssectional interpolation of annual rings within a $3 \mathrm{D}$ root model. Dendrochronologia, 29, 201-210.

Wu Q (2016). Analyzing and Modeling of Root Architecture of Individual Maize Plants Based on High-accuracy Field Data and Its Applications. PhD dissertation, China Agricultural University, Beijing. [吴茜 (2016). 基于精确数 据的大田玉米植株根系结构分析、建模及其应用. 博士 学位论文, 中国农业大学, 北京.]
Wu Q, Pagès L, Wu J (2016). Relationships between root diameter, root length and root branching along lateral roots in adult, field-grown maize. Annals of Botany, 117, 379.

Xu GQ, Li Y (2008). Rooting depth and leaf hydraulic conductance in the xeric tree Haloxyolon ammodendron growing at sites of contrasting soil texture. Functional Plant Biology, 35, 1234-1242.

Yan XL, Liao H, Ge ZY, Luo XW (2000). Root architectural characteristics and phosphorus acquisition efficiency in plants. Chinese Bulletin of Botany, 17, 511-519. [严小龙, 廖红, 戈振扬, 罗锡文 (2000). 植物根构型特性与磷吸 收效率. 植物学通报, 17, 511-519.]

Yang M, Défossez P, Danjon F, Fourcaud T (2018). Analyzing key factors of roots and soil contributing to tree anchorage of Pinus species. Trees, 32, 703-712.

Yu P, Hochholdinger F, Li C (2015). Root-type-specific plasticity in response to localized high nitrate supply in maize (Zea mays). Annals of Botany, 116, 751-762.

Zhao YY, Lu ZH, Xia JB, Liu JT (2015). Root architecture and adaptive strategy of 3 shrubs in Shell Bay in Yellow River Delta. Acta Ecologica Sinica, 35, 1688-1695. [赵艳云，陆 兆华, 夏江宝, 刘京涛 (2015). 黄河三角洲贝壳堤岛 3 种优势灌木的根系构型. 生态学报, 35, 1688-1695.]

责任编委: 王政权 责任编辑: 李 敏 